\title{
Folded Acoustic Phonon Modes in Ge/Si Quantum Dot Superlattices With Different Periods
}

\author{
Zheng Yang ${ }^{1 *}$, Jian-Lin Liu ${ }^{1}$, Yi Shi ${ }^{2}$, You-Dou Zheng ${ }^{2}$, and Kang L. Wang ${ }^{3}$ \\ ${ }^{1}$ Quantum Structures Laboratory, Department of Electrical Engineering, \\ University of California at Riverside, Riverside, California 92521, USA \\ ${ }^{2}$ Department of Physics, Nanjing University, Nanjing 210093, China \\ ${ }^{3}$ Department of Electrical Engineering, University of California at Los Angeles, \\ Los Angeles, California 90095, USA
}

\begin{abstract}
(Received 01 July 2005; accepted 26 February 2006)
We present low-frequency Raman scattering measurements on self-assembled Ge/Si quantum dot superlattice samples grown by molecular beam epitaxy. The samples had different growth parameters, such as the number of periods, nominal thicknesses of Ge layers, and growth temperatures. Raman scattering peaks were observed in the low-frequency region $\left(<30 \mathrm{~cm}^{-1}\right)$, which were attributed to the folded acoustic phonon modes related to the periodicity of the Ge/Si quantum dot superlattices. We applied Rytov's model to calculate the frequencies of the folded acoustic phonons in the $\mathrm{Ge} / \mathrm{Si}$ quantum dot superlattices. The calculated frequencies were in good accordance with the experimental results among most of the samples. It was observed from the experiments that the Raman peak intensities decreased with the increased order of the folded acoustic phonons. Raman peak intensities from different order folded acoustic phonons were explained by photoelastic effect theory. It was also found that the intensities of Raman peaks were related to the Ge layer thickness and the periods of the $\mathrm{Ge} / \mathrm{Si}$ quantum dot superlattices: the thinner the Ge layer thickness, the lower the intensity; the smaller the periods, the lower the intensity of the Raman peaks.
\end{abstract}

\section{INTRODUCTION}

In recent years, self-assembled $\mathrm{Ge} / \mathrm{Si}$ quantum dot superlattices (QDSLs) were found to have promising applications in optoelectronics and thermoelectrics, such as photodetectors and novel thermoelectric devices etc. Towards these goals, the properties of the electrons and phonons in such kind of $\mathrm{Ge} / \mathrm{Si}$ nanostructures were investigated by photoluminescence ${ }^{1-3}$ and Raman scattering $^{4-18}$ techniques.

The nonresonant and resonant Raman scattering measurements in self-assembled Ge/Si QDSLs were first reported by Liu et al. ${ }^{4}$ and Kowk et al. ${ }^{5}$, respectively. Then, most of the published Raman studies on Ge/Si QDSLs were focused on the optical phonons. ${ }^{6,}{ }^{9-14}$ It was demonstrated that valuable information about strain and $\mathrm{Si} / \mathrm{Ge}$ interdiffusion in the QDSLs could be derived from the GeGe optical phonon mode (around $300 \mathrm{~cm}^{-1}$ ) in the Raman spectra. ${ }^{9-14}$ Few studies on Raman scattering by acoustic

*Author to whom correspondence should be addressed. phonons in self-assembled Ge/Si QDSLs were reported until Liu et al. observed a series of peaks in the range from 60 to $150 \mathrm{~cm}^{-1}$ in the Raman spectra. ${ }^{6}$ Then, several explanations were considered in Yu's comment ${ }^{7}$ on this work and in the response of Liu et al. ${ }^{8}$. Milekhin et al. observed a series of doublet peaks below $100 \mathrm{~cm}^{-1}$ in the Raman spectra of Ge/Si QDSLs, ${ }^{9}$ which were attributed to the folded longitudinal acoustic (LA) phonons in the superlattices and explained by the Rytov's model. ${ }^{19}$ Raman spectra of $\mathrm{Ge} / \mathrm{Si}$ QDSLs in the range below $60 \mathrm{~cm}^{-1}$ have also been observed by Milekhin et al., ${ }^{15}$ Cazayous et al., ${ }^{16,17}$ and Tan et al., ${ }^{18}$ in both resonant and nonresonant Raman scattering modes. However, few publications have reported on the low-frequency Raman spectra from a series of samples with different number of periods and structural data, especially in nonresonant Raman scattering mode, partly due to the fact that the signals were rather weak. In this article, we report the systematic study of the nonresonant low-frequency Raman spectra of self-assembled Ge/Si QDSLs in a series of samples with different number of periods and Ge layer thicknesses. 
Table 1. Growth parameters and structural data of the 10 samples.

\begin{tabular}{lcccc}
\hline & $\begin{array}{c}\text { Nominal } \\
\text { thickness } \\
\text { of Ge layer } \\
d_{2}(\mathrm{~nm})\end{array}$ & $\begin{array}{c}\text { Nominal } \\
\text { thickness } \\
\text { of Si spacer } \\
\text { layer } d_{1}(\mathrm{~nm})\end{array}$ & $\begin{array}{c}\text { Growth } \\
\text { temperature } \\
\left({ }^{\circ} \mathrm{C}\right)\end{array}$ & $\begin{array}{c}\text { Periods } \\
N\end{array}$ \\
\hline $\mathrm{A}$ & 0.6 & 20 & 600 & 22 \\
$\mathrm{~B}$ & 1.2 & 20 & 600 & 22 \\
$\mathrm{C}$ & 1.5 & 20 & 600 & 22 \\
$\mathrm{D}$ & 1.2 & 20 & 540 & 10 \\
$\mathrm{E}$ & 1.5 & 20 & 540 & 10 \\
$\mathrm{~F}$ & 1.5 & 20 & 540 & 2 \\
$\mathrm{G}$ & 1.5 & 20 & 540 & 5 \\
$\mathrm{H}$ & 1.5 & 20 & 540 & 20 \\
$\mathrm{I}$ & 1.5 & 20 & 540 & 35 \\
$\mathrm{~J}$ & 1.5 & 20 & 540 & 50 \\
\hline
\end{tabular}

\section{EXPERIMENTAL}

Ten samples, labeled A to J, were grown by a solid-source PerkinElmer molecular beam epitaxy (MBE) system with Stranski-Kranstanow (S-K) growth mode on Si (100) substrate. For each of the 10 samples, a 100-nm thick Si buffer layer was deposited on the substrate, followed by some identical $\mathrm{Ge} / \mathrm{Si}$ bilayers to form superlattices. Each bilayer included a Ge layer and a Si spacer layer with the nominal thickness of $20 \mathrm{~nm}$. No Si cap layer was used in the samples.

These 10 samples had different nominal thicknesses of Ge layers, the number of periods, and the growth temperatures. The nominal thicknesses of the $\mathrm{Ge}$ layer in samples A, B, and C were $0.6,1.2$, and $1.5 \mathrm{~nm}$, respectively, and all three samples were of 22 periods and grown at $600^{\circ} \mathrm{C}$. Samples D and E were both 10-period samples grown at $540^{\circ} \mathrm{C}$, in which the nominal thicknesses of the Ge layer were 1.2 and $1.5 \mathrm{~nm}$, respectively. Samples F, G, $\mathrm{H}$, I, and $\mathrm{J}$ were grown at $540^{\circ} \mathrm{C}$ with the same nominally thickness 1.5 -nm Ge layer and consisted of 2, 5, 20, 35 , and 50 periods, respectively. The growth parameters and structural data of the 10 samples are summarized in Table 1. Raman scattering measurements were performed with a JY T64000 Raman system in backscattering configuration at room temperature. All the spectra were excited by the 514-nm line of an $\mathrm{Ar}^{+}$laser and recorded with a liquid-nitrogen-cooled charge coupled device (CCD) camera. The spectra were obtained using the same excitation power and data accumulation time. The spectra resolution is about $0.7 \mathrm{~cm}^{-1}$. The beginning frequency point of the Raman spectra is around $8 \mathrm{~cm}^{-1}$.

\section{RESULTS}

Figure 1 shows the Raman spectrum of sample E. The spectrum can be distinctly divided into two regimes; one is the high-frequency region $\left(250-550 \mathrm{~cm}^{-1}\right)$, which corresponds to the optical phonon modes in the Ge/Si QDSLs samples, and the other is the low-frequency region $\left(<30 \mathrm{~cm}^{-1}\right)$ of acoustic modes. The $\mathrm{Ge}-\mathrm{Ge}, \mathrm{Si}-\mathrm{Ge}$, and $\mathrm{Si}-$ Si peaks located at 301,421 , and $521 \mathrm{~cm}^{-1}$, respectively,

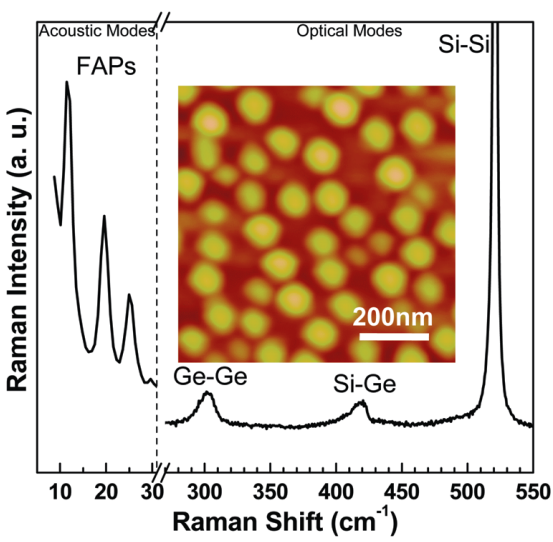

Fig. 1. Raman spectrum of sample E. Raman peaks from FAPs and optical phonon modes appeared in the low- and high-frequency regions, respectively, which were detached by the dashed vertical line. The inset shows the AFM image of sample E in a $1 \times 1 \mu \mathrm{m}^{2}$ area.

are attributed to $\mathrm{Ge}, \mathrm{SiGe}$ alloys, and $\mathrm{Si}$ substrate in the samples. The Raman peaks of the low-frequency region arise from the folded acoustic phonon (FAP) modes in the Ge/Si QDSLs. The inset of Figure 1 shows the atomic force microscopy (AFM) image of sample E. The density and average base size of the quantum dots are about $4 \times 10^{9} \mathrm{~cm}^{-2}$ and $0.1 \mu \mathrm{m}$, respectively.

Figure 2 shows the cross-sectional transmission electron microscopy (TEM) images of the 10-, 20-, and 35-period self-assembled Ge/Si QDSLs samples (i.e., samples E, H, and I). The dark lens-shaped areas, the dark stripes, and bright contrast belts in the TEM images are Ge quantum dots, Ge wetting layers, and $\mathrm{Si}$ spacer layers, respectively. The vertical correlations of the Ge quantum dots were clearly seen from the images. From the images, it can be observed that the actual effective thickness of $\mathrm{Si}$ spacer tayers has shrunk to a smaller value than the nominal thickness $(20 \mathrm{~nm})$ since the $\mathrm{Si}$ spacers were deposited on the curvature surfaces of three-dimensional dots.

Figure 3a shows the Raman spectra of samples A, B, and $\mathrm{C}$ and an identical $\mathrm{Si}$ substrate. Since the nominal thickness of the Ge layer in sample A is too thin to form any $\mathrm{Ge}$ quantum dots, $\mathrm{A}$ is only a superlattice sample consisting of $\mathrm{Ge}$ wetting layers and $\mathrm{Si}$ spacer layers but without quantum dots. B and C are 22-period superlattice samples with Ge quantum dots. As seen from Figure 3a, the Raman scattering peaks can be clearly found at 16 and $15 \mathrm{~cm}^{-1}$ for samples B and C, respectively, and no obvious Raman scattering peaks in sample $\mathrm{A}$ and $\mathrm{Si}$ substrate. In addition, the peak observed in sample $\mathrm{C}$ is stronger than that of sample B. These low-frequency Raman scattering peaks originate from the FAPs in the samples related to the periodicity of the superlattices. No low-frequency Raman scattering peak was found in the Si substrate because of its lack of periodicity. For sample A, the Ge layers in the superlattice were too thin; therefore, Raman peaks were too weak to be observed, as analyzed here. 


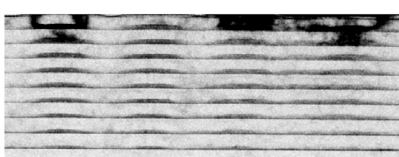

(a) $100 \mathrm{~nm}$

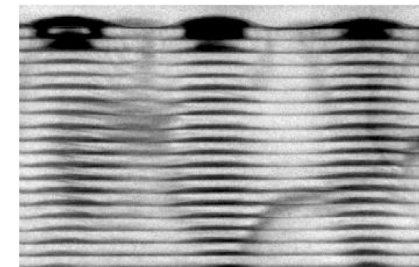

(b)

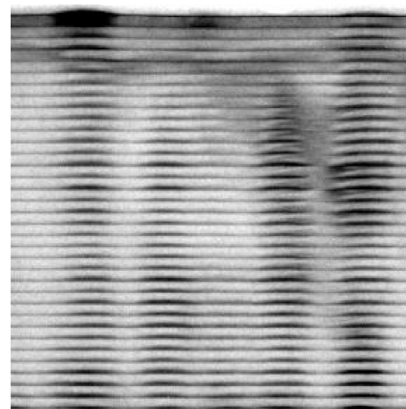

(c)

$100 \mathrm{~nm}$

Fig. 2. Cross-sectional TEM images of Ge/Si QDSL samples (a) E, (b) H, and (c) I, which included 10-, 20-, and 35-period Ge/Si bilayers, respectively. The nominal thicknesses of Si spacer and Ge layers were 20 and $1.5 \mathrm{~nm}$, respectively. The Ge quantum dots were vertically correlated in the superlattices.

Figure $3 \mathrm{~b}$ shows the Raman spectra of samples D and E and an identical Si substrate. D and E are 10-period QDSL samples with different nominal thicknesses of Ge layers. Three obvious Raman peaks were found in both samples $\mathrm{D}$ and E, locating at 11,19 , and $25 \mathrm{~cm}^{-1}$ and 11,20 , and $25 \mathrm{~cm}^{-1}$, respectively. The Raman peaks of sample E are
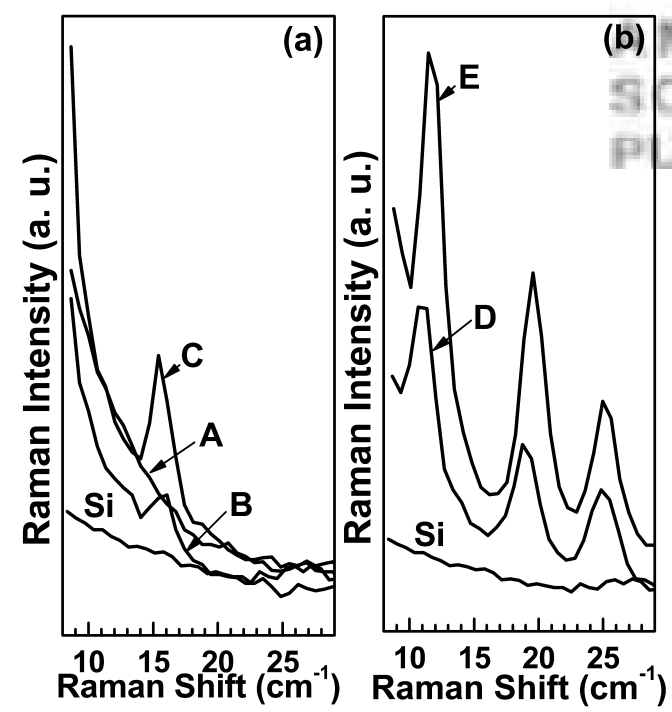

Fig. 3. Low-frequency Raman spectra of samples (a) A, B, C, and an identical Si substrate and (b) D, and $\mathrm{E}$ and an identical $\mathrm{Si}$ substrate. Sample A is a sample without quantum dots that just has Ge wetting layers in the superlattice, while sample B, C, D, and E are superlattice samples with quantum dots.

relatively stronger than those of sample D. The three peaks of both samples decreased step by step from low to high frequency.

Figure 4 shows the low-frequency Raman scattering spectra of a series of samples, F to J, that have the same nominal thickness of Ge layers but a different number of superlattice periods. The Raman scattering spectrum of an identical Si substrate is also shown. Samples F, G, H, I, and $\mathrm{J}$ have 2, 5, 20, 35, and 50 periods of Ge/Si bilayers, respectively. From Figure 4, three Raman peaks were found in samples $\mathrm{H}$, I, and $\mathrm{J}$, and only one peak was observed in sample G. The peak positions are presented in the left half of Table 2. No peak was seen from sample $\mathrm{F}$ and $\mathrm{Si}$ substrate. It was found that the intensities of the Raman peaks increased with the number of the periods of superlattices. Sample F has just 2 periods; the Raman peaks are too weak to be observed. Sample G has 5 periods, and only one peak was found. The intensities of Raman scattering peaks from samples H, I, and J increased with their superlattice periods, and the three Raman peaks in each of the samples decreased with the FAPs' incremental order.

6 The inset of Figure 4 shows the relations between the normalized Raman intensities and number of superlattice periods of samples $\mathrm{H}, \mathrm{I}$, and $\mathrm{J}$ from three different orders. In the graph, Raman peak intensity of first-order FAPs in sample $\mathbf{J}$ was taken as unit, and all other peak intensities were normalized by this value. Nonresonant Raman

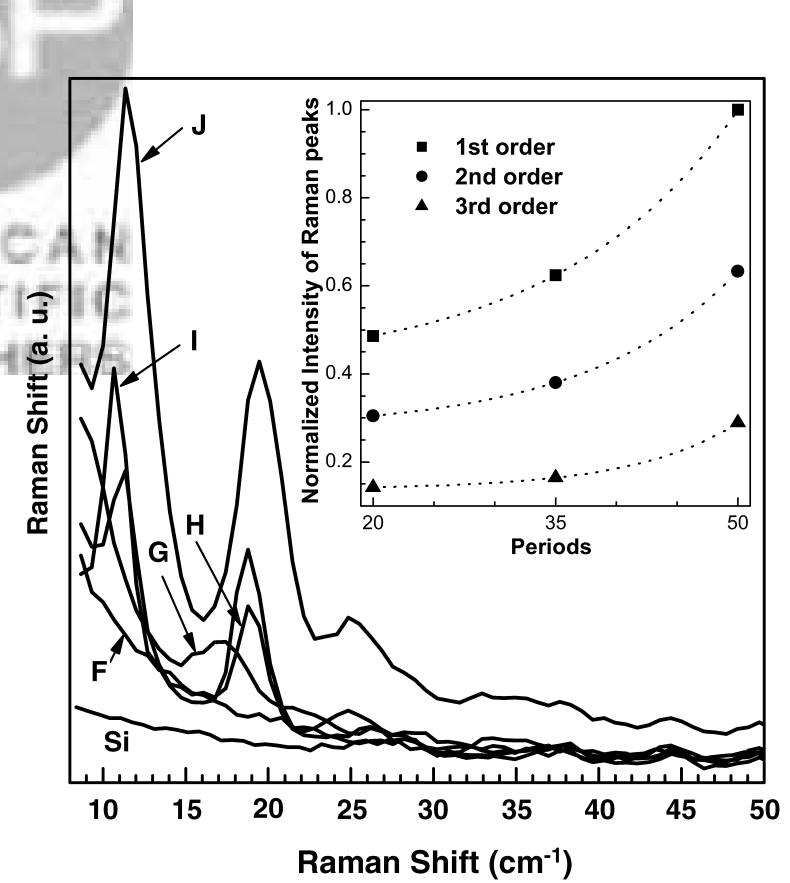

Fig. 4. Low-frequency Raman spectra of the samples F, G, H, I, J, and an identical Si substrate. Samples F, G, H, I, and J include 2, 5, 20, 35, and 50 periods of $\mathrm{Ge} / \mathrm{Si}$ bilayers, respectively. The inset shows the relation between the normalized intensities of the Raman peaks from the first-, second-, and third-order FAPs and the number of the periods of the superlattice samples $\mathrm{H}$, I, and $\mathrm{J}$. 
Table 2. Experimental and calculated Raman frequencies of the first four orders of FAPs.

\begin{tabular}{|c|c|c|c|c|c|c|c|c|}
\hline \multirow[b]{3}{*}{ Sample } & \multicolumn{4}{|c|}{$\begin{array}{c}\text { Experimental FAP } \\
\text { Frequencies } \omega_{\exp }\left(\mathrm{cm}^{-1}\right)\end{array}$} & \multicolumn{4}{|c|}{$\begin{array}{c}\text { Calculated FAP } \\
\text { Frequencies } \omega_{\text {cal }}\left(\mathrm{cm}^{-1}\right)\end{array}$} \\
\hline & \multicolumn{4}{|c|}{$n$} & \multicolumn{4}{|c|}{$m$} \\
\hline & 0 & 1 & 2 & 3 & 0 & $1-$ & $1+$ & $2-$ \\
\hline A & - & - & - & - & 4.4 & 11.8 & 20.7 & 28.1 \\
\hline B & - & - & 16.1 & - & 4.4 & 11.2 & 20.0 & 26.8 \\
\hline $\mathrm{C}$ & - & - & 15.4 & - & 4.4 & 10.9 & 19.6 & 26.1 \\
\hline D & - & 10.7 & 18.8 & 24.8 & 4.4 & 11.2 & 20.0 & 26.8 \\
\hline $\mathrm{E}$ & - & 11.5 & 19.6 & 25.0 & 4.4 & 10.9 & 19.6 & 26.1 \\
\hline $\mathrm{F}$ & - & - & - & - & 4.4 & 10.9 & 19.6 & 26.1 \\
\hline G & - & - & 17.4 & - & 4.4 & 10.9 & 19.6 & 26.1 \\
\hline $\mathrm{H}$ & - & 11.4 & 18.8 & 26.2 & 4.4 & 10.9 & 19.6 & 26.1 \\
\hline I & - & 10.7 & 18.8 & 24.8 & 4.4 & 10.9 & 19.6 & 26.1 \\
\hline $\mathrm{J}$ & - & 11.4 & 19.5 & 24.8 & 4.4 & 10.9 & 19.6 & 26.1 \\
\hline
\end{tabular}

scattering from FAPs in superlattices can be considered as a coherent sum of scattering within different layers. ${ }^{20}$ Thus, the superlattice samples with more periods have correspondingly larger Raman intensities, which was in accordance with the experimental results. $\bigcirc u$ Ou experimental results showed that the intensities appear in an exponential relation with the superlattice periods. Some interesting theoretical works on how the Raman scattering spectra of the Ge QDSLs depend on the quantum dot sizes and the number of quantum dot layers have already been reported by Cazayous et al., ${ }^{17}$ but there were few detailed experimental reports on several kinds of Ge/Si QDSLs samples with superlattice period number and structural data before our work, especially the experiments in nonresonant Raman scattering mode.

\section{DISCUSSION}

The reason for these low-frequency periodic acoustic phonon peaks is associated with a superlattice for which low-frequency zone-edge phonon modes have been folded into the zone center rather than phonon confinement in quantum dots. ${ }^{21}$ Rytov's elastic continuum model has been applied to explain the experimental results, in which the acoustic phonon dispersions of Ge/Si QDSLs were written as $^{19}$

$$
\begin{aligned}
\cos (q d)= & \cos \left(\frac{\omega d_{1}}{V_{1}}\right) \cos \left(\frac{\omega d_{2}}{V_{2}}\right) \\
& -\frac{k^{2}+1}{2 k} \sin \left(\frac{\omega d_{1}}{V_{1}}\right) \sin \left(\frac{\omega d_{2}}{V_{2}}\right)
\end{aligned}
$$

where $d_{1}$ and $d_{2}, V_{1}$ and $V_{2}, \rho_{1}$ and $\rho_{2}$ are thicknesses, sound velocities, and densities of the $\mathrm{Si}$ spacer and $\mathrm{Ge}$ layers, respectively; $q$ is the superlattice wave vector; $d=$ $d_{1}+d_{2}$ is the superlattice period; and $k=V_{1} \rho_{1} /\left(V_{2} \rho_{2}\right)$ is a ratio coefficient. For large $d$ and frequencies less than $100 \mathrm{~cm}^{-1}$, the dispersion is assumed to be linear, and the FAP dispersion can be approximately simplified $\operatorname{as}^{22,} 23$

$$
\omega \cong \frac{1}{2 \pi c}\left(\frac{2 m \pi}{d} \pm q\right) V_{s}
$$

where $c$ is the speed of light; $m=0,1,2, \ldots$ is the folding index of the FAPs; and $V_{\mathrm{S}}$ is the acoustic velocity in the superlattice, which is given in Rytov's theory as

$$
V_{s}=d\left(\frac{d_{1}^{2}}{V_{1}^{2}}+\frac{d_{2}^{2}}{V_{2}^{2}}+\left(k+\frac{1}{k}\right) \frac{d_{1} d_{2}}{V_{1} V_{2}}\right)^{-1 / 2}
$$

The scattering wave vector $q_{\mathrm{S}}$ in the backscattering geometry can be roughly determined as

$$
q_{s} \cong \frac{4 \eta(\lambda) \pi}{\lambda}\left(1-\frac{1}{4 \eta^{2}(\lambda)}\right)
$$

with $\lambda$ and $\eta(\lambda)$ the incident laser light wavelength and the refractive index of the material at that wavelength, respectively. We approximately consider the refractive indexes of both $\mathrm{Si}$ and $\mathrm{Ge}$ as $\eta \approx 4.2$ for $\lambda=514 \mathrm{~nm}$. Thus, we employ this value throughout the superlattices (i.e., for both Ge layers and Si spacer layers).

The densities of $\mathrm{Si}$ and $\mathrm{Ge}$ are 2.3 and $5.3 \mathrm{~g} / \mathrm{cm}^{3}$, respectively, and the sound velocities in pure $\mathrm{Si}$ and $\mathrm{Ge}$ layers are 8.4 and $4.9 \mathrm{~km} / \mathrm{s}$, respectively. For the Si spacer layers composed of pure $\mathrm{Si}$, the density and sound velocity were directly employed with values of $\mathrm{Si}$, that is, $\rho_{1}=$ $\rho(\mathrm{Si})=2.3 \mathrm{~g} / \mathrm{cm}^{3}$, and $V_{1}=8.4 \mathrm{~km} / \mathrm{s}$. In the Ge layers of Ge/Si QDSLs samples, however, the Ge quantum dots and wetting layers are not pure $\mathrm{Ge}$ but the alloy of $\mathrm{Ge}$ and $\mathrm{Si}$ due to the $\mathrm{Si} / \mathrm{Ge}$ interdiffusion during the growth. The composition of $\mathrm{Ge}$ can be estimated from the intensity ratio among the Raman peaks in the optical region. ${ }^{10-14}$

The Ge compositions of the quantum dots were calculated as around $50 \%$ in our samples. ${ }^{11}$ Then, the sound velocity in Ge layers can be obtained by the linear interpolation of sound velocity of pure $\mathrm{Si}$ and $\mathrm{Ge}$. We assumed the density and sound velocity of Ge layers as following: $\rho_{2}=$ $\left[\rho(\mathrm{Si})+\rho(\mathrm{Ge}) / 2=3.8 \mathrm{~g} / \mathrm{cm}^{3}\right.$, and $V_{2}=[V(\mathrm{Si})+V(\mathrm{Ge}) / 2=$ $6.7 \mathrm{~km} / \mathrm{s}$.

The designed thickness of the Si spacer layer for all the samples was $20 \mathrm{~nm}$. But, as described, the actual thicknesses of the Si spacer layers were shrunk due to the existence of $\mathrm{Ge}$ quantum dots. For different samples, the shrunken values were slightly different as characterized by cross-sectional TEM images. We employed an approximately general value $d_{1}=16.5 \mathrm{~nm}$ for all the Si spacers in the following calculations. In addition, we took the nominal thicknesses of the Ge layers as values of $d_{2}$, which was also employed in Refs. 9 and 15 . The $d_{2}$ values of different samples are listed in Table 1.

Figure $5 \mathrm{~b}$ shows the calculated dispersions of FAPs in sample E. The vertical dashed line in Figure $5 b$ stands for the scattering wave vector in the experiments. The three 


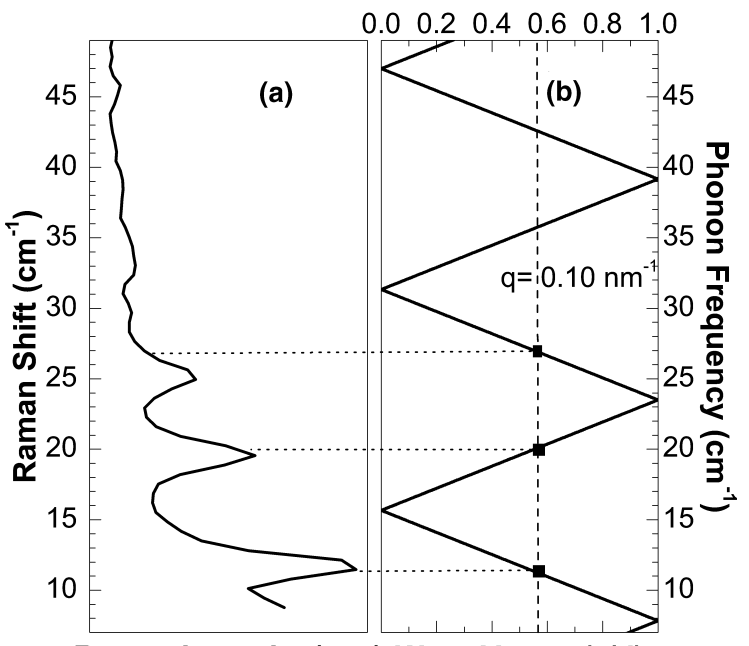

Raman Intensity (a.u.) Wave Vector ( $\pi / d)$

Fig. 5. (a) Experimental results of Raman scattering and (b) calculated dispersions of FAPs in sample E. The dashed vertical line stands for the scattering wave vector $q_{s}$. The three solid intersections stand for the calculated frequencies of FAPs. The dotted horizontal lines are used to facilitate the comparison between experimental results and calculations.

intersections between the solid FAP dispersion curve and the dashed line are the frequencies of FAPs with three different orders. The frequencies of the FAPs are in good accordance with the experimental results. The calculated frequencies of the FAPs for all the samples are listed in the right half of Table 2. The calculated zero-order FAP was not observed in the experiments due to the relatively high initial point frequency $\left(8 \mathrm{~cm}^{-1}\right)$ in the Raman spectra. The FAPs from first to third order observed in the experiments (listed in the left side of Table 2) agree with the calculations. The FAPs above fourth order were not observed in our experiments due to the relatively low intensities compared with the previous orders.

The Raman intensity $I_{n}$ of the $n$ th-order FAPs can be estimated based on photoelastic effect as ${ }^{20,24}$

$$
I_{n} \propto \frac{\sin ^{2}\left(n \pi d_{2} / d\right)}{n^{2}}\left[\omega_{n}\left(b_{n}+1\right)\right]
$$

where $\omega_{n}$ is the frequency of $n$ th-order FAPs in the Ge/Si QDSLs, and $b_{n}$ is their Bose factor. The Raman spectra of samples D and E shown in Figure $3 \mathrm{~b}$ show that sample $\mathrm{E}$ has a relatively higher Raman intensity than sample D. This can be roughly explained by Eq. (5). Since sample E has a larger $d_{2}$ value $(1.5 \mathrm{~nm})$ than that of sample $\mathrm{D}$ $(1.2 \mathrm{~nm})$, it has a relatively larger Raman intensity for the same order FAPs. The peak intensity difference between samples $\mathrm{C}$ and $\mathrm{B}$ can also be explained in the same way. In the same sample, the intensities of FAP peaks decreased with the increase of the order. This is also in accordance with the theory [Eq. (5)]. We fitted the relation of peak intensities and orders using square $\operatorname{Sinc}(n)$ functions, which were shown as solid lines in Figure 6. The symbols in Figure 6 are the experimental results of Raman peak
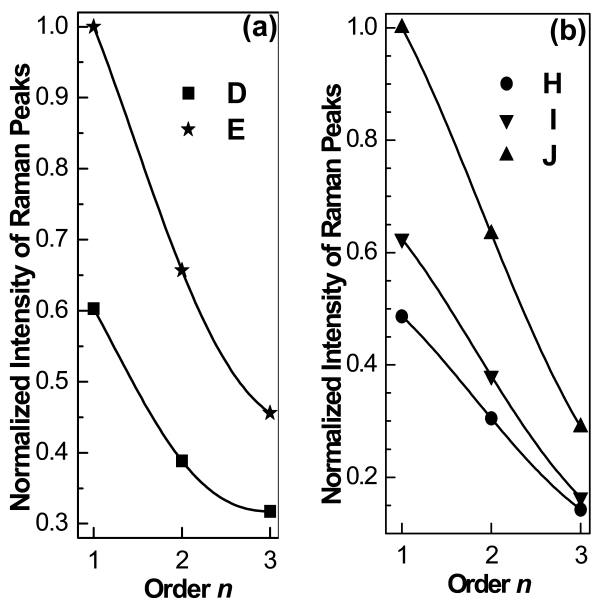

Fig. 6. Experimental results and fitted curves of different normalized intensities of the Raman peaks on the first three order FAPs in (a) samples $\mathrm{D}$ and $\mathrm{E}$ and (b) samples H, I, and J. The symbols in the graphs are the experimental results, and the lines are the fitted curves.

Ingenta to:

intensities from the first three order FAPs in different samples, which were normalized by the first-order peak intensities of sample $\mathrm{E}$ in Figure $6 \mathrm{a}$ and $\mathrm{J}$ in Figure $6 \mathrm{~b}$, respectively. The trend of the symbols follows the solid lines well.

\section{CONCLUSIONS}

In summary, we have reported the low-frequency Raman scattering spectra of self-assembled Ge/Si QDSLs. Lowfrequency Raman scattering peaks arose from the FAPs in the Ge/Si QDSLs. Rytov's model was applied to calculate the frequencies of the FAPs in the Ge/Si QDSLs. The calculated frequencies were in good accordance with the experimental results among most of the samples. The Raman peak intensities decreased with the increasing order of FAPs. Raman peak intensities from different order FAPs have been fitted, and the intensities of the Raman peaks were related to the Ge layer thickness and the periods of the Ge/Si QDSLs: the thinner the Ge layer thickness, the lower the intensity; the smaller the periods, the lower the intensity of the Raman peaks.

Acknowledgments: The work at the University of California at Riverside was supported by DARPA/DMEA through CNID, and we greatly acknowledge Dr. S. G. Thomas of Motorola and Dr. Q. H. Xie of Freescale Semiconductor for TEM characterizations. The work at Nanjing University was supported by science funds of China under grants 60225014 and 60390074.

\section{References}

1. J. Wan, G. Jin, Z. M. Jiang, Y. H. Luo, J. L. Liu, K. L. Wang, X. Z. Liao, and J. Zou. Appl. Phys. Lett., 79, 1980 (2001).

2. M. W. Dashiell, U. Denker, C. Müller, G. Costantini, C. Manzano, K. Kern, and O. G. Schmidt. Appl. Phys. Lett., 80, 1279 (2002). 
3. Z. Yang, Y. Shi, J. L. Liu, B. Yan, R. Zhang, Y. D. Zheng, and K. L. Wang. Mater. Lett., 58, 3765 (2004).

4. J. L. Liu, Y. S. Tang, K. L. Wang, T. Radetic, and R. Gronsky. Appl. Phys. Lett., 74, 1863 (1999).

5. S. H. Kwok, P. Y. Yu, C. H. Tung, Y. H. Zhang, M. F. Li, C. S. Peng, and J. M. Zhou. Phys. Rev. B, 59, 4980 (1999).

6. J. L. Liu, G. Jin, Y. S. Tang, Y. H. Luo, K. L. Wang, and D. P. Yu. Appl. Phys. Lett., 76, 586 (2000).

7. P. Y. Yu. Appl. Phys. Lett., 78, 1160 (2001).

8. J. L. Liu, G. Jin, Y. S. Tang, Y. H. Luo, K. L. Wang, and D. P. Yu. Appl. Phys. Lett., 78, 1162 (2001).

9. A. G. Milekhin, N. P. Stepina, A. I. Yakinmov, A. I. Nikiforov, S. Schulze, and D. R. T. Zahn. Euro. Phys. J., B16, 355 (2000).

10. M. Cazayous, J. Groenen, F. Demangeot, R. Sirvin, M. Caumont, T. Remmele, M. Albrecht, S. Christiansen, M. Becher, H. P. Strunk, and H. Wawra. J. Appl. Phys., 91, 6772 (2002).

11. J. L. Liu, J. Wan, Z. M. Jiang, A. Khitun, K. L. Wang, and D. P. Yu. J. Appl. Phys., 92, 6804 (2002).

12. P. H. Tan, K. Brunner, and D. Bougeard, and G. Abstreiter. Phys. Rev. B, 68, 125302 (2003).

13. Z. Yang, Y. Shi, J. L. Liu, B. Yan, Z. X. Huang, L. Pu, Y. D. Zheng, and K. L. Wang. Chin. Phys. Lett., 20, 2001 (2003).
14. A. V. Baranov, A. V. Fedorov, T. S. Perova, R. A. Moore, S. Solosin, V. Yam, D. Bouchier, and V. Le Thanh. J. Appl. Phys., 96, 2857 (2004).

15. A. G. Milekhin, A. I. Nikiforov, O. P. Pchelyakov, S. Schulze, and D. R. T. Zahn. Nanotechnology, 13, 55 (2002).

16. M. Cazayous, J. Groenen, J. R. Huntzinger, A. Mlayah, and O. G. Schmidt. Phys. Rev. B, 64, 033306 (2001).

17. M. Cazayous, J. Groenen, A. Zwick, A. Mlayah, R. Carles, J. L. Bischoff, and D. Dentel. Phys. Rev. B, 66, 195320 (2002).

18. P. H. Tan, D. Bougeard, G. Abstreiter, and K. Brunner. Appl. Phys. Lett., 84, 2632 (2004).

19. S. M. Rytov. Akoust. Zh., 2, 71 (1956).

20. C. Colvard, T. A. Gant, M. V. Klein, R. Merlin, R. Fischer, H. Morkoc, and A. C. Gossard. Phys. Rev. B, 31, 2080 (1985).

21. J. L. Liu, A. Khitun, and K. L. Wang, in Dekker Encyclopedia of Nanoscience and Nanotechnology, J. A. Schwarz, C. I. Contescu, and K. Putyera, Eds., Marcel Dekker, New York (2004), pp. 3203-3211.

22. D. J. Lockwood, M. W. C. Dharma-wardana, J.-M. Baribeau, and D. C. Houghton. Phys. Rev. B, 35, 2243 (1987).

23. P. X. Zhang, D. J. Lockwood, H. J. Labbé, and J.-M. Baribeau. Phys. Rev. B, 46, R9881 (1992).

24. H. Brugger, G. Abstreiter, H. Jorke, H. J. Herzog, and E. Kasper. Phys. Rev. B, 33, R5928 (1986).

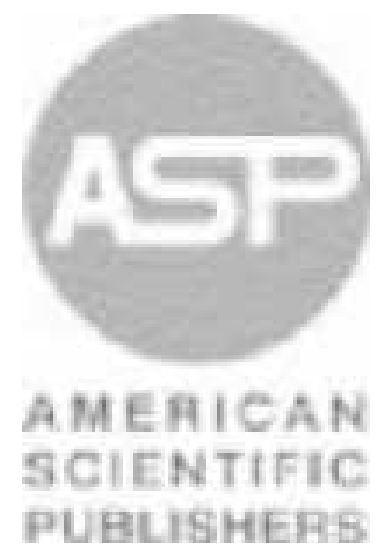

